\title{
Une carrière hospitalière est-elle encore motivante pour les médecins?
}

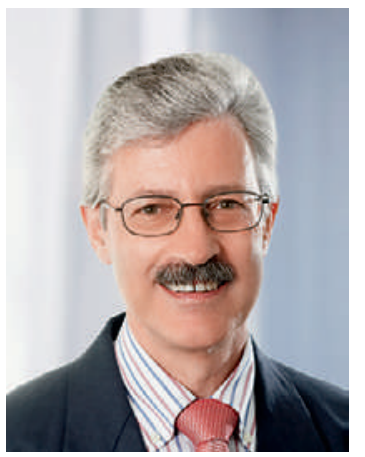

Il y a quelque temps, les premiers pas de l'étudiant en médecine à l'hôpital le mettaient en contact avec une hiérarchie forte, dont la tête paraissait inaccessible. Frâ̂chement diplômé, l'assistant débutant ne ressentait pas les choses différemment. C'était l'époque du mandarinat, avec des médecins chefs tout-puissants au caractère souvent excessif et distribuant généreusement les châtiments, parfois même physiques dans les milieux chirurgicaux. Détenant l'essentiel du pouvoir dans l'institution, ces figures de proue étaient néanmoins adulées par leurs subordonnés qui supportaient ce régime pénitentiaire sans broncher, avec l'espoir de gravir eux-mêmes les échelons pour pouvoir occuper, à leur tour, une telle position enviée.

En deux décennies, tout a fondamentalement changé. Les médecins hospitaliers ont dû céder de leur influence au profit des soignants et des responsables administratifs. Les assistants et chefs de clinique ont acquis des droits et ont passé du statut de forces de travail corvéables à merci à celui de collaborateurs respectés. Ainsi, les médecins chefs d'aujourd'hui ne peuvent plus être des patrons à l'ancienne mais doivent mener leur service avec charisme, tact et entregent tout en faisant preuve de bonnes capacités de gestion.

\section{Le pouvoir et l'argent sont-ils les facteurs essentiels de la motivation pour une carrière hospitalière?}

Pour ceux qui ont étudié la médecine afin de soigner les patients, il n'est pas évident de se mettre à disposition pour occuper un poste à responsabilité avec ses nouvelles contingences. Longtemps, la fonction de médecin cadre s'est vue honorée par de confortables revenus. Dans tout le pays, ceux-ci ont cependant drastiquement diminué au cours des années. Particulièrement dans le secteur public, le libéralisme n'est plus de mise, avec des conditions générales de rémunération de plus en plus contraignantes et restrictives. Paradoxalement, quand il y a perte d'une certaine liberté d'un côté, on assiste de l'autre à des tentatives de réintroduire la variabilité des honoraires par plusieurs formes de salaire au mérite, sur le modèle de ce qui se pratique dans le monde de l'industrie et de la finance (voir à ce propos l'article de B. Meyer à la page 1381).

Le pouvoir et l'argent sont-ils les facteurs essentiels de la motivation à embrasser une carrière hospitalière? Si cela a pu être le cas jadis, ce n'est plus du tout d'actualité. L'hôpital est un formidable milieu de travail où les interactions humaines sont riches et nombreuses, que ce soit avec les patients ou avec les collaborateurs de l'établissement. La pluridisciplinarité s'y rencontre tous les jours, avec une très haute interactivité entre les protagonistes. La formation y est omniprésente, au niveau prégradué aussi bien que postgradué: la transmission du savoir aux générations suivantes est une tâche noble et enthousiasmante, garantissant aussi le maintien du niveau de ses propres connaissances au contact permanent de jeunes collègues curieux et informés des derniers développements scientifiques. Dans sa discipline, le médecin cadre hospitalier

\section{Quand il y a perte d'une certaine}

\section{liberté d'un côté, on assiste de l'autre}

à des tentatives de réintroduire

la variabilité des honoraires.

assure la couverture des meilleures prestations au profit des malades de sa région, en bonne intelligence avec les praticiens référents. Il a ainsi une responsabilité vis-à-vis du système et de la société.

Progressivement, la pression économique augmente, rendant nécessaire une gestion analytique avec priorisation des besoins. Effectuée avec objectivité, une telle démarche n'est pas rebutante pour les médecins mais bien plutôt constructive et fédératrice, incitant à améliorer les comportements et les processus.

Une carrière hospitalière reste donc, en 2013, très motivante pour les disciples d'Hippocrate. Plus que la rétribution qui doit être adéquate, l'ambiance et la solidarité qui règnent dans un établissement représentent l'alchimie susceptible d'entretenir l'attrait des postes médicaux à responsabilité, pour lesquels des candidats sont encore prêts à s'investir avec passion sans compter leur temps ni leur énergie.

Dr Pierre-François Cuénoud, vice-président de la FMH, responsable du domaine Tarifs et économie de la santé pour les médecins hospitaliers 